MODELING, IDENTIFICATION AND CONTROL, 1996, vOL. 17, NO. 2, 107-119

doi:10.4173/mic.1996.2.4

\title{
A globally stable adaptive ship autopilot with wave filter using only yaw angle measurements
}

\author{
TRYGVE LAUVDAL $\dagger$ and THOR I. FOSSEN $\dagger$
}

Keywords: Global stability, adaptive ship autopilot, output feedback, notch filtering, integral action, velocity observer.

\begin{abstract}
A stable minimum phase transfer function from rudder angle to yaw angle is used to design a globally stable adaptive ship autopilot. First-order wave disturbances in yaw are filtered by applying a notch filter. Integral action is introduced by using a reference model technique. Global stability is proven for the total system which include the yaw rate observer, the parameter update law, the feedback controller, the notch filter and the integral part of the controller. The simulation results showed that the performance is excellent, even with no a priori knowledge of the ship parameters.
\end{abstract}

\section{Introduction}

This paper addresses the problem of controlling a ship with unknown parameters by using feedback only from the yaw angle. In addition the problem of high-frequency rudder motions due to 1st-order wave disturbances in the feedback loop is addressed. This problem is usually solved by using a Kalman-filter to estimate the low-frequency motion components of the ship and use these in the controller. Wave filtering in terms of the Kalman filter algorithm has been discussed by Balchen et al. (1976, 1980a, b), Grimble et al. (1980a, b), Sælid and Jenssen (1983), Reid et al. (1984) and Holzhüter and Strauch (1987). However, if the wave filter, the control law and the parameter update law are designed independently, global stability and robustness cannot be guaranteed. In this paper an unified approach based on cascaded notch filtering and Model Reference Adaptive Control (MRAC) is proposed. The MRAC scheme is based on the input error direct adaptive control law of Bodson (1986) and Bodson and Sastry (1987). This scheme uses observers instead of full state feedback (see Fig. 1) and the reference model has to be stable and of the same relative degree as the plant.

This approach proved to have several advantages; only measurement of the yaw angle is necessary and in theory it is possible to filter the 1st order wave disturbances sufficiently and prove global stability at the same time. In addition no exact tuning of the initial values of the control parameters was necessary. Low-frequency disturbances due to currents and wind were compensated for by adding integral action in the controller. This is done by modifying the reference model. Finally, the controlled ship showed to be robust and globally stable also under rudder saturation. This is due to the fact that the input error is used to update the parameter estimates.

Received 20 August 1995.

The Norwegian Institute of Technology, Department of Engineering Cybernetics, N-7034 Trondheim, Norway. (E-mail: lauvdal@itk.unit.no, tif@itk.unit.no).

Presented at the 3rd IFAC Workshop on Control Applications in Marine Systems, Trondheim, Norway, May 1995 (CAMS '95). 


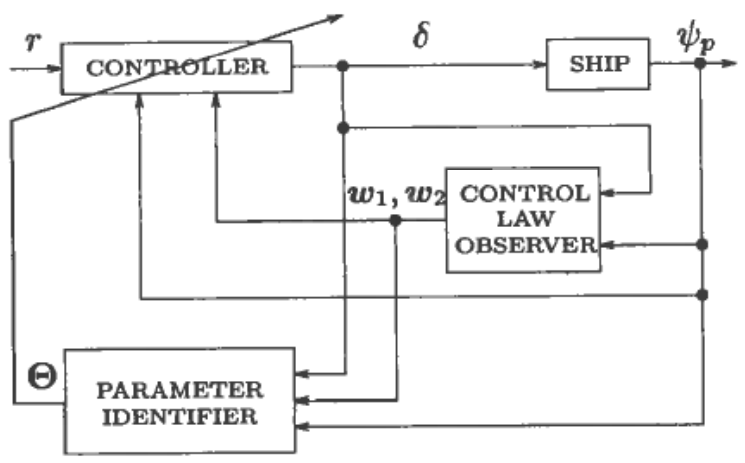

Figure 1. Block diagram showing MRAC principle.

\section{Direct adaptive control: an input error approach}

The first step is to derive an adaptive control law under the assumption that the ship is minimum phase. This constraint is no limiting factor, since all ships are minimum phase from rudder $(\delta)$ to yaw angle $\left(\psi_{p}\right)$.

Consider the ship model of Nomoto et al. (1957)

$$
\left[\begin{array}{c}
\dot{v} \\
\dot{r} \\
\dot{\psi}_{p}
\end{array}\right]=\left[\begin{array}{ccc}
a_{11} & a_{12} & 0 \\
a_{21} & a_{22} & 0 \\
0 & 1 & 0
\end{array}\right]\left[\begin{array}{c}
v \\
r \\
\psi_{p}
\end{array}\right]+\left[\begin{array}{c}
b_{1} \\
b_{2} \\
0
\end{array}\right] \delta
$$

where $\psi_{p}$ is the yaw angle, $r$ is the angular velocity in yaw and $v$ is the sway velocity. Applying the Laplace transformation to this model yields a 3rd-order transfer function

$$
\frac{\psi_{p}}{\delta}(s)=\frac{\left(1+T_{1} s\right)}{s\left(1+T_{2} s\left(1+T_{3} s\right)\right.}
$$

For simplicity eqn. (2) is written as

$$
\frac{\psi_{p}}{\delta}(s)=P(s) \triangleq K_{p} \frac{n_{p}(s)}{d_{p}(s)}
$$

where the polynomials $n_{p}(s)$ and $d_{p}(s)$ have degrees $m=1$ and $n=3$, respectively, and therefore $P(s)$ has relative degree $\gamma_{p}=2$. These polynomials are also monic and coprime because $T_{1} \neq T_{2}$ and $T_{1} \neq T_{3}$.

\subsection{Reference model}

A reference model with dynamic properties equal to the desired closed-loop ship dynamics has to be chosen. A natural choice is a 2nd-order critical damped system

$$
\frac{\psi_{m}}{r}(s)=\frac{\omega_{0}^{2}}{s^{2}+2 \zeta \omega_{0} s+\omega_{0}^{2}}
$$

where $\zeta=1$ and $\omega_{0}$ is the natural frequency. The transfer function is

$$
\frac{\psi_{m}}{r}(s)=M(s) \bumpeq K_{m} \frac{n_{m}(s)}{d_{m}(s)}
$$

with obvious choices of $n_{m}(s)$ and $d_{m}(s)$. Notice that the reference model has relative degree $\gamma_{m}=\gamma_{p}=2$. 


\subsection{Output feedback law}

The control design methology of Bodson (1986) and Bodson and Sastry (1987), see also Sastry and Bedson (1989), is applied to design a globally stable output feedback autopilot. Consider the control law

$$
\delta(s)=c_{0} r(s)+\frac{c(s)}{\lambda(s)} \delta(s)+\frac{d^{\prime}(s)}{\lambda(s)} \psi_{p}(s)
$$

where $d^{\prime}(s) / \lambda(s) \psi_{p}(s)$ can be written as:

$$
\frac{\delta^{\prime}(s)}{\lambda(s)} \psi_{p}(s)=d_{0} \psi_{p}(s)+\frac{d(s)}{\lambda(s)} \psi_{p}(s)
$$

Here $\lambda(s)$ is chosen equal to $\lambda_{0}(s) n_{m}(s)$, whereas $n_{m}(s)$ is the numerator of the reference model. The only constraint is that $\lambda_{0}(s)$ must be Hurwitz. In addition the polynomials have to satisfy $\operatorname{dim}(\hat{\lambda})=\mathrm{n}-1, \operatorname{dim}(\mathrm{c})=\mathrm{n}-2$ and $\operatorname{dim}(\mathrm{d})=\mathrm{n}-2$. Moreover

$$
\begin{aligned}
& \lambda(s)=s^{2}+\lambda_{2} s+\lambda_{1} \\
& c(s)=c_{2} s+c_{1} \\
& d(s)=d_{2} s+d_{1}
\end{aligned}
$$

The control law given by Eqns. (6) and (7) is linear in its parameters. This can be seen by defining two signals

$$
\begin{aligned}
& w_{1}(s) \triangleq \frac{1}{\lambda(s)}\left[\begin{array}{l}
1 \\
s
\end{array}\right] \delta(s) \\
& w_{2}(s) \triangleq \frac{1}{\lambda(s)}\left[\begin{array}{l}
1 \\
s
\end{array}\right] \psi_{p}(s)
\end{aligned}
$$

Hence

$$
\begin{aligned}
& \frac{c(s)}{\lambda(s)} \psi_{p}(s)=c^{\mathbf{T}} \boldsymbol{w}_{1}(s) \\
& \frac{d(s)}{\lambda(s)} \psi_{p}(s)=\boldsymbol{d}^{\mathrm{T}} \boldsymbol{w}_{2}(s)
\end{aligned}
$$

where $\boldsymbol{c}^{\mathrm{T}}=\left[c_{1}, c_{2}\right]$ and $\boldsymbol{d}^{\mathrm{T}}=\left[d_{1}, d_{2}\right]$. Hence the control law (6) and (7) can be written:

$$
\delta(t)=\boldsymbol{\Theta}^{\mathrm{T}}(t) \boldsymbol{w}(t)
$$

where

$$
\begin{aligned}
& \boldsymbol{\Theta}(t) \triangleq\left[c_{0}(t), \boldsymbol{c}^{\mathrm{T}}(t), d_{0}(t), \boldsymbol{d}^{\mathrm{T}}(t)\right]^{\mathrm{T}} \\
& \boldsymbol{w}(t) \triangleq\left[r(t), \boldsymbol{w}_{1}^{\mathrm{T}}(t), \psi_{p}(t), \boldsymbol{w}_{2}^{\mathrm{T}}(t)\right]^{\mathrm{T}}
\end{aligned}
$$

Notice that the controller parameters are time-varying. This is because an identifier algorithm will be applied to estimate $\Theta(t)$, see Section 2.3.

If the ship dynamics is known, the exact control parameters can be calculated from the closed-loop identity

$$
\frac{c_{0} K_{p} \lambda(s) n_{p}(s)}{[\lambda(s)-c(s)] d_{p}(s)-K_{p} n_{p}(s) d^{\prime}(s)}=M(s)
$$


which has the unique solution

$$
\begin{aligned}
c_{0}^{*} & =\frac{K_{m}}{K_{p}} \\
c^{*}(s) & =\lambda(s)-q(s) n_{p}(s) \\
d^{\prime *}(s) & =\frac{1}{K_{p}}\left[q(s) d_{p}(s)-\lambda_{0}(s) d_{m}(s)\right]
\end{aligned}
$$

when $\gamma_{p}=\gamma_{m}$. Then, application of the control law

$$
\delta(t)=\boldsymbol{\Theta}^{* \mathrm{~T}} \mathrm{w}(t)
$$

to the ship model (2) makes the closed-loop dynamics equal to the reference model dynamics (4).

\subsection{Identifier structure}

Equation (5) can be written

$$
r(s)=M^{-1}(s) \psi_{m}(s)
$$

Hence the plant input can be defined as

$$
r_{p}(s) \cong M^{-1}(s) \psi_{p}(s)
$$

Then the input error $e_{i}(s)$ can be defined as

$$
\begin{aligned}
e_{i}(s) & \triangleq r_{p}(s)-r(s) \\
& =M^{-1}(s)\left(\psi_{p}(s)-\psi_{m}(s)\right)
\end{aligned}
$$

Perfect tracking, see Eqn. (16), implies that it is possible to derive an expression for the input error

$$
e_{i}(s)=\frac{1}{c_{0}(s)} \Phi^{\mathrm{T}}(s) z(s)
$$

where

$$
z(s)=\left[r_{p}(s) \bar{w}^{\mathrm{T}}(s)\right]^{\mathrm{T}} \quad, \Phi(s)=\Theta(s)-\Theta^{*}
$$

and

$$
\overline{\boldsymbol{w}}(s)=\left[\boldsymbol{w}_{1}^{\mathrm{T}}(s), \psi_{p}(s), \boldsymbol{w}_{2}^{\mathrm{T}}(s)\right]^{\mathrm{T}}
$$

The signal $r_{p}(s)=M^{-1}(s) \psi_{p}(s)$ is not available since $\gamma_{m}=2$. Hence a modification of the input error is necessary. Therefore a new transfer function, $L^{-1}(s)$, is introduced. This transfer function is stable, minimum phase and has relative degree $\gamma_{l}=n-m=2$. Moreover, the following signal can be defined

$$
\begin{aligned}
v(s) & \triangleq L^{-1}(s) z(s) \\
& =\left[\begin{array}{c}
(M(s) L(s))^{-1} \psi_{p}(s) \\
L^{-1}(s) \bar{w}(s)
\end{array}\right]^{\mathrm{T}}
\end{aligned}
$$

Notice that the signal $(M(s) L(s))^{-1} \psi_{p}(s)$ is available since $M(s) L(s)$ has relative degree $\gamma_{m l}=0$. This leads to the definition of the modified input error

$$
\begin{aligned}
e_{2}(s) & =\boldsymbol{\Phi}^{\mathrm{T}}(s) v(s) \\
& =\boldsymbol{\Theta}^{\mathrm{T}}(s) \boldsymbol{v}(s)-L^{-1}(s) \delta(s)
\end{aligned}
$$

where $e_{2}(s) \in \mathbb{R}$. 


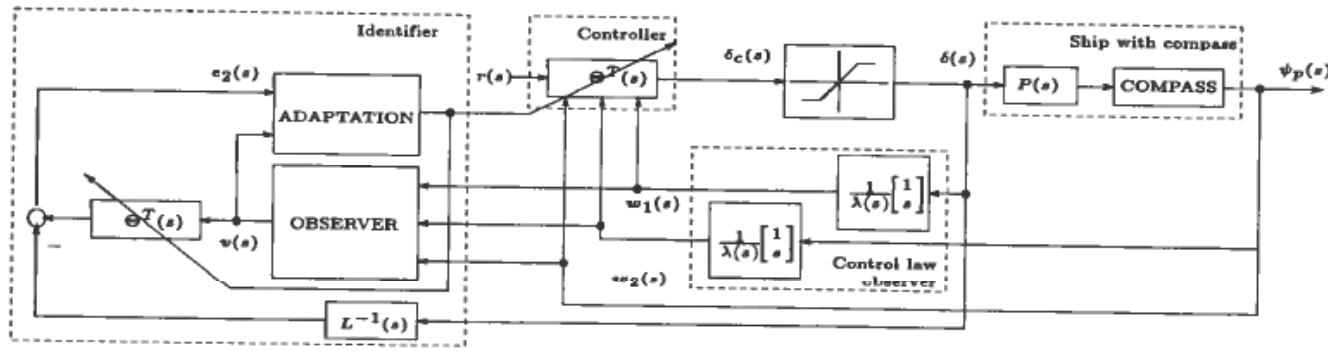

Figure 2. Block diagram showing MRAC applied to a ship with yaw measurements.

\subsection{Identification algorithm}

Given the modified input error in Eqn. (25), a identification algorithm is used to update the control parameters in Eqn. (13). Because the controller is linear in its parameters several algorithms are available. For instance, the normalized least square algorithm

$$
\begin{gathered}
\dot{\boldsymbol{\Theta}}(t)=-g \frac{e_{2}(t) \boldsymbol{P}(t) \boldsymbol{v}(t)}{1+\gamma \boldsymbol{v}^{\mathrm{T}}(t) \boldsymbol{P}(t) \boldsymbol{v}(t)} \\
\dot{\boldsymbol{P}}(t)=-g \frac{\boldsymbol{P}(t) \boldsymbol{v}(t) \boldsymbol{v}^{\mathrm{T}}(\mathrm{t}) \boldsymbol{P}(t)}{1+\gamma \boldsymbol{v}^{\mathrm{T}}(t) \boldsymbol{P}(t) \boldsymbol{v}(t)}
\end{gathered}
$$

with $g, \gamma>0$ and $\boldsymbol{P}(t) \in \mathbb{R}^{6 \times 6}$ showed excellent performance. The ship with the MRAC is shown in Fig. 2.

\subsection{Integral action}

It is necessary to introduce integral action to avoid steady-state errors due to slowly varying disturbances like currents, wave drift and wind. Notice that the reference model (4) can be written

$$
M(s)=\frac{h_{0}(s)}{1+h_{0}(s)}
$$

where

$$
h_{0}(s)=\frac{\omega_{0}^{2}}{s\left(s+2 \zeta \omega_{0}\right)}
$$

is the reference model open-loop transfer function. Hence integral action can be obtained by defining a modified open-loop transfer function

$$
\tilde{h}_{0}(s)=\frac{1+T_{i} s}{T_{i} s} h_{0}(s)
$$

which yields the following reference model

$$
\tilde{M}(s)=\omega_{0}^{2} \frac{\left(s+1 / T_{i}\right)}{s^{3}+2 \zeta \omega_{0} s^{2}+\omega_{0}^{2} s+\omega_{0}^{2} / T_{i}}
$$

From Eqn. (30) it is easy to see that the reference model (31) has integral action, and therefore the ship will obtain the same property. 


\subsection{Stability analysis}

If a gain $K_{\max }$ is defined, such that

$$
K_{p} \leqslant K_{\max }
$$

then, according to Appendix B, the ship (3) with reference model (4) or (31), controller (13), identifier structure (25) and identification algorithm (26) is globally stable. In addition $e_{2}(t) \rightarrow 0$ and thus $\psi_{p}(t) \rightarrow \psi_{m}(t)$ whereas $\Phi(t)$ is bounded. Rudder saturation does not affect this result.

\section{Adaptive control law with cascaded notch filter}

An important part of a ship autopilot is the wave filter. The wave filter is included to avoid high-frequency rudder motions. This is usually solved by applying a Kalman-filter to estimate the low-frequency motion components. In this section another approach is suggested. The ship model is augmented with a notch filter and the augmented model is used for control design. The augmented ship model is

$$
\begin{aligned}
\bar{P}(s)=B(s) P(s) & =K_{p} K_{b} \frac{n_{b}(s) n_{p}(s)}{d_{b}(s) d_{p}(s)} \\
& =\bar{K}_{p} \frac{\bar{n}_{p}(s)}{d_{p}(s)}
\end{aligned}
$$

where $\bar{K}_{p}=K_{p} K_{b}, \bar{n}_{p}(s)=n_{b}(s) n_{p}(s)$ and $\bar{t}_{p}(s)=d_{b}(s) d_{p}(s)$, see Fig. 3. $B(s)$ is a notch filter given by

$$
B(s)=\frac{\left(s^{2}+2 \zeta_{e} \omega_{e} s+\omega_{e}^{2}\right)^{k}}{\left(s+\omega_{e}\right)^{2 k}}
$$

where $\omega_{e}$ is the encounter frequency and $\zeta_{e} \ll 1$. Notice that $\bar{K}_{p}=k_{p} K_{b}=\mathrm{K}_{\mathrm{p}}$. The augmented ship model can be written as

$$
\psi_{p}^{L}(s)=B(s) P(s) u(s)=B(s) \psi_{p}(s)
$$

where $\psi_{p}^{L}(s)$ is the low frequency part of $\psi_{p}(s)$ which is used for feedback. Therefore, high-frequency rudder motions can be avoided by proper selection of $B(s)$.

\subsection{Reference model}

Notice that the relative degree is unchanged, that is $\gamma_{\bar{p}}=\gamma_{p}$. Hence augmentation of the reference model is not necessary, and $\bar{M}(s)=M(s)$.

\subsection{Adaptive control law with notch filter}

From (6) and (7), the following polynomials are obtained

$$
\begin{aligned}
& \lambda(s)=s^{2 k+2}+\lambda_{2 k+2} s^{2 k+1}+\ldots+\lambda_{1} \\
& c(s)=c_{2 k+2} s^{2 k+1}+\ldots+c_{1} \\
& d(s)=d_{2 k+2} s^{2 k+1}+\ldots+d_{1}
\end{aligned}
$$


Then, by defining

$$
\begin{gathered}
\boldsymbol{w}_{1}(s) \triangleq \frac{1}{\lambda(s)}\left[\begin{array}{c}
1 \\
\vdots \\
s^{2 k+1}
\end{array}\right] \delta(s) \\
\boldsymbol{w}_{2}(s) \triangleq \frac{1}{\lambda(s)}\left[\begin{array}{c}
1 \\
\vdots \\
s^{2 k+1}
\end{array}\right] \psi_{p}(s)
\end{gathered}
$$

the control law

$$
\delta(t)=\Theta(t)^{\mathrm{T}} w(t)
$$

is given by

$$
\begin{aligned}
c(t) & =\left[c_{1}(t), c_{2}(t), \ldots, c_{2 k+2}(t)\right]^{\mathrm{T}} \\
d(t) & =\left[d_{1}(t), d_{2}(t), \ldots, d_{2 k+2}(t)\right]^{\mathrm{T}} \\
\Theta(t) & =\left[c_{0}(t), c^{\mathrm{T}}(t), d_{0}(t), d^{\mathrm{T}}(t)\right]^{\mathrm{T}} \\
w(t) & =\left[r(t), w_{1}^{\mathrm{T}}(t), \psi_{p}(t), w_{2}^{\mathrm{T}}(t)\right]^{\mathrm{T}}
\end{aligned}
$$

\subsection{Identifier structure}

The identifier structure is now given by

$$
e_{2}(s)=\boldsymbol{\Theta}^{\mathrm{T}}(s) v(s)-L^{-1}(s) \delta(s)
$$

where $L^{-1}(s)$ is a stable, minimum phase transfer function with relative degree $\lambda_{I}=n-m$ and

$$
\boldsymbol{v}(s)=L^{-1}(s)\left[\begin{array}{c}
M^{-1}(s) \psi_{p}(s) \\
\boldsymbol{w}_{1}(s) \\
\psi_{p}(s) \\
\boldsymbol{w}_{2}(s)
\end{array}\right]
$$

\subsection{Identification algorithm}

The identification algorithm for Eqns. (44) and (45) is given by

$$
\begin{gathered}
\dot{\boldsymbol{\Theta}}(t)=-g_{1} \frac{e_{2}(t) \boldsymbol{P}(t) \boldsymbol{v}(t)}{1+\gamma \boldsymbol{v}^{\mathrm{T}}(t) \boldsymbol{P}(t) \boldsymbol{v}(t)} \\
\dot{\boldsymbol{P}}(t)=-g_{1} \frac{\boldsymbol{P}(t) \boldsymbol{v}(t) \boldsymbol{v}^{\mathrm{T}}(t) \boldsymbol{P}(t)}{1+\gamma \boldsymbol{v}^{\mathrm{T}}(t) \boldsymbol{P}(t) \boldsymbol{v}(t)}
\end{gathered}
$$

where $g, \gamma>0$ and $\boldsymbol{P}(t) \in \mathbb{R}^{(4 k+6) \times(4 k+6) \text {. }}$

The ship with the notch filter $B(s)$ and MRAC is shown in Fig. 3 . 


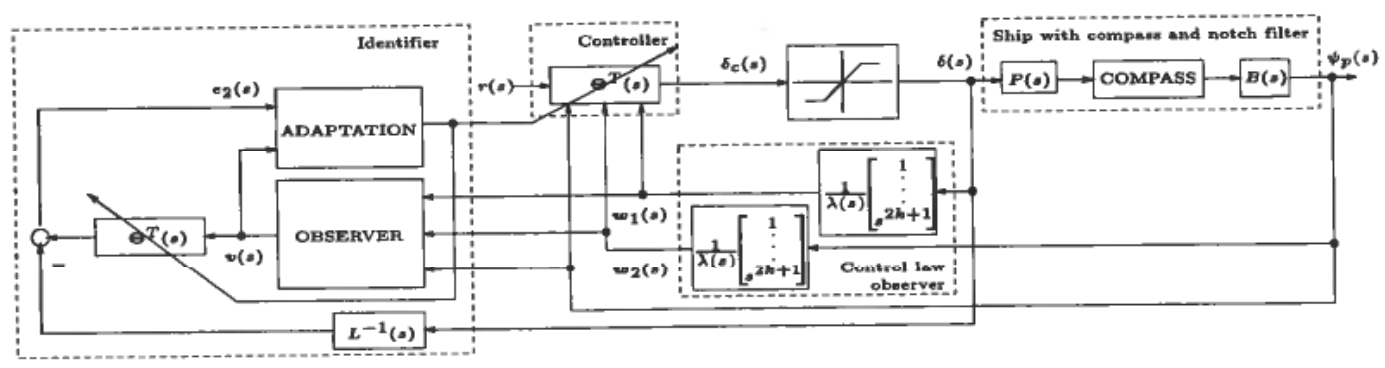

Figure 3. Block diagram showing the MRAC and the notch filter.

\subsection{Stability analysis}

Again, if a gain $K_{\max }$ is defined, such that

$$
K_{p} \leqslant K_{\max }
$$

then, according to Appendix B, the ship (3) with reference model (4) or (31), controller (39), identifier structure (44) and identification algorithm (46) is globally stable. In addition $e_{2}(t) \rightarrow 0$ and thus $\psi_{p}^{L}(t) \rightarrow \psi_{m}(t)$ whereas $\boldsymbol{\Phi}(t)$ is bounded. Rudder saturation does not affect this result.

\section{Simulation study of the adaptive ship autopilot}

A simulation study is done for the ship model presented in Appendix A. The desired ship dynamics is given by (4) whereas the parameters were chosen as

$$
\omega_{0}=0 \cdot 05, \quad \zeta=1
$$

The initial parameter vector and weighting matrix are chosen as

$$
\begin{aligned}
\Theta(0) & =\left[c_{0}, c^{\mathrm{T}}(0), 0, d^{\mathrm{T}}(0)\right]^{\mathrm{T}} \\
c(0) & =d(0)=\mathbf{0} \\
\mathbf{P}(0) & =\boldsymbol{I}
\end{aligned}
$$

and the adaptation parameters are chosen as

$$
g=\gamma=1 \cdot 0
$$

The transfer function $L^{-1}(s)$ is chosen to be equal the reference model

$$
L^{-1}(s)=M(s)
$$

The sample frequency is $1(\mathrm{~Hz})$.

\section{Case 1: PD control}

Figure 4 shows the time-response of the ship when

$$
\lambda(s)=\lambda_{0}(s)=(s+0.05)^{2}
$$

Notice that after only one step in the reference, the closed-loop dynamics matches the reference model. 


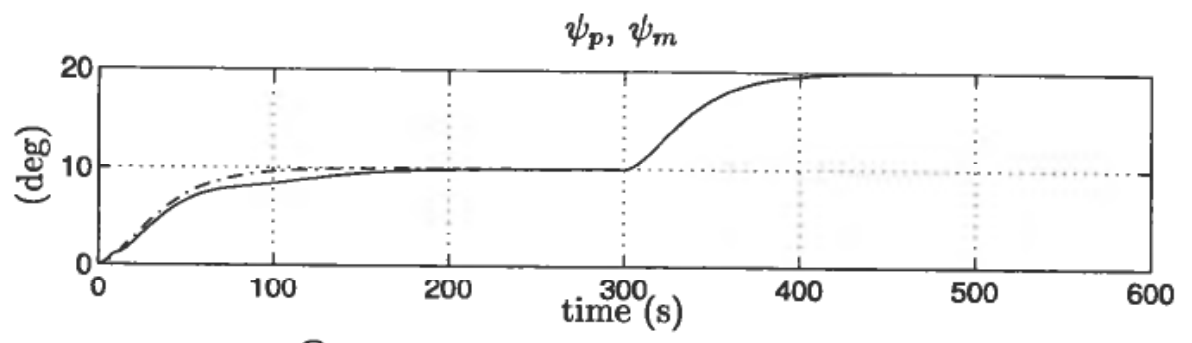

$\Theta$
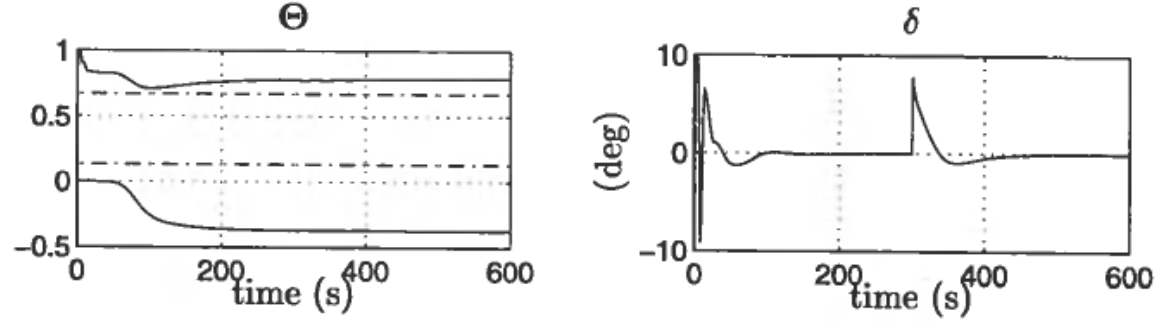

Figure 4. The plots show the measurcd yaw angle $\psi_{p}$ and the desired yaw angle $\psi_{m}$ (dotted) for a step input $r=10(\mathrm{deg})$ when $t=0(\mathrm{~s})$ and $r=20(\mathrm{deg})$ when $t=300(\mathrm{~s})$. In addition the time response for the parameter estimates $c_{0}$ and $d_{0}$ (the dotted lines indicate the exact values, $c_{0}^{*}$ and $d_{0}^{*}$ ) and the rudder $\delta$ are shown.

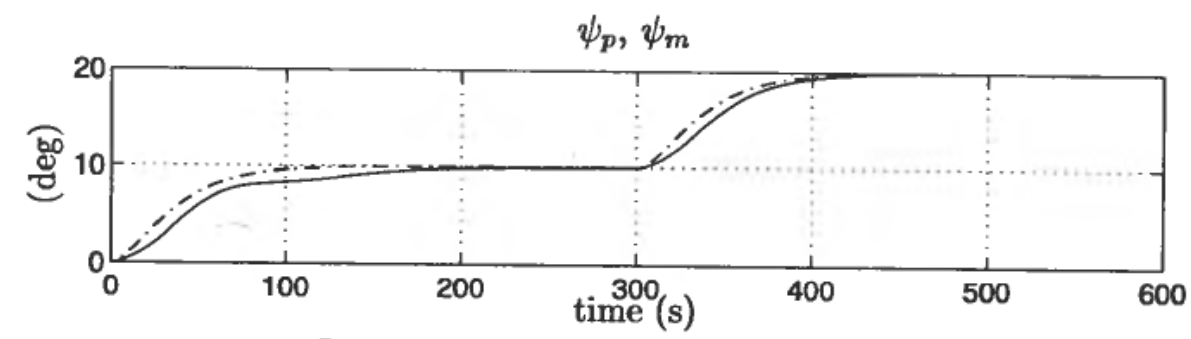

$\Theta$
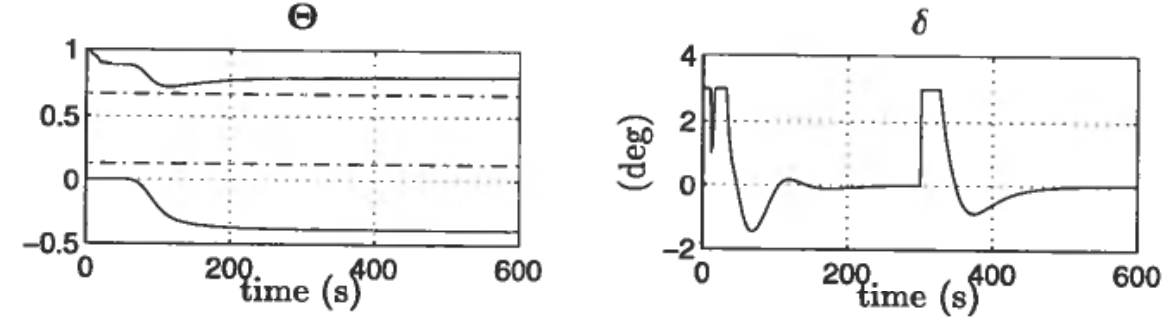

Figure 5. The plots show the measured yaw angle $\psi_{p}$ and the desired yaw angle $\psi_{m}$ (dotted) for a step input $r=10(\mathrm{deg})$ when $t=0$ (s) and $r=20$ (deg) when $t=300$ (s). In addition the time response for the parameter estimates $c_{0}$ and $d_{0}$ (the dotted lines indicate the exact values, $c_{0}^{*}$ and $\left.d_{0}^{*}\right)$ and the rudder $\delta$ are shown. Notice that the rudder saturates $\left(\delta_{\max }=3\right.$ (deg)) without affecting the stability.

\section{Case 2: PD-control with rudder limitation}

Figure 5 shows the time response when $\delta_{\max }=3(\mathrm{deg})$ and the rudder saturates. Notice that rudder saturation do not influence the stability. The performance is, however, somewhat reduced. 
Case 3: PD-control with wave filtering

Figure 6 shows the time response of the ship when wave filtering is included by using the notch filter in (34) with

$$
k=1, \zeta_{e}=0.01, \omega_{e}=0.7
$$

In addition

$$
\lambda(s)=\lambda_{0}(s)=(s+1)^{2}(s+0 \cdot 1)^{2}
$$

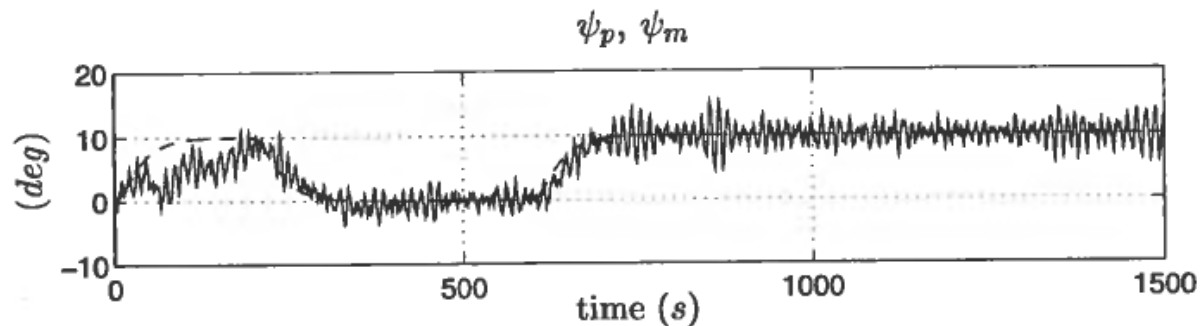

$\Theta$

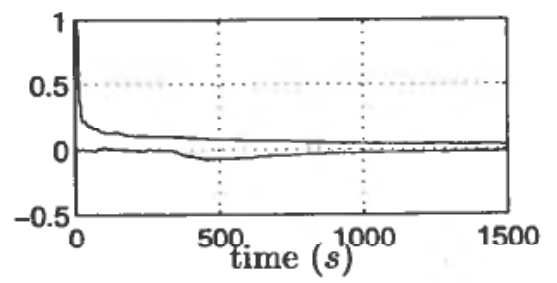

$\delta$

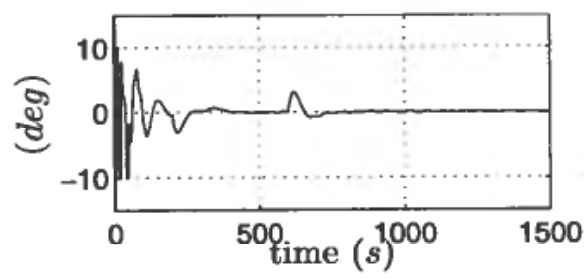

Figure 6. The plots show the measured yaw angle $\psi_{p}$ and desired yaw angle $\psi_{m}$ (dotted) for a step input, $r=10(\mathrm{deg})$ when $t=0(\mathrm{~s})$ and $t=600(\mathrm{~s})$ and $r=0(\mathrm{deg})$ when $t=200(\mathrm{~s})$, when there are lst-order wave disturbances (max value $\pm 3(\mathrm{deg})$ ) on the yaw angle measurement. In addition the time response for the parameter estimates $c_{0}$ and $d_{0}$ and the rudder $\delta$ is shown.

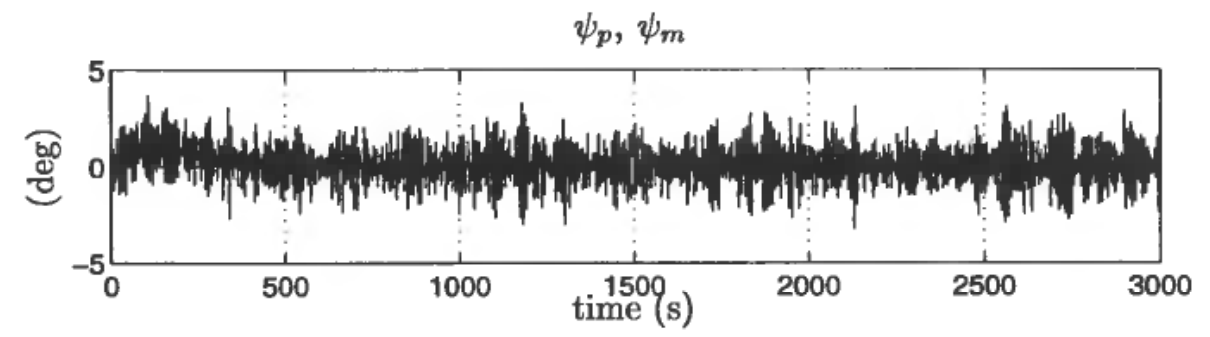

$\Theta$

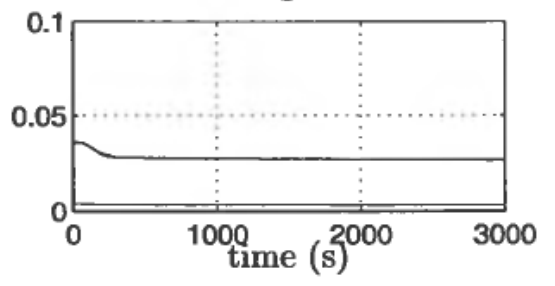

$\delta$

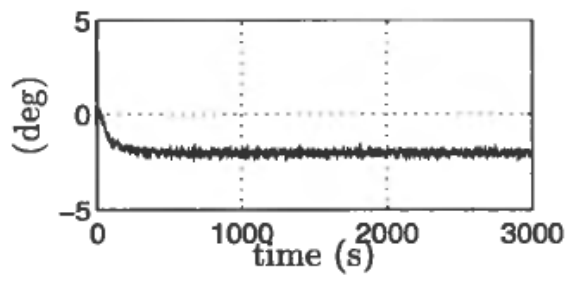

Figure 7. The plots show the measured yaw angle $\psi_{p}$ and desired yaw angle $\psi_{m}=0$ (dotted) for $r=0$ (deg). In addition there are 1st-order wave disturbances (max value $\pm 3(\mathrm{deg})$ ) on the yaw measurements and a offset of $2(\mathrm{deg})$ in the rudder. The time response for the parameter estimates $c_{0}, d_{0}$ and the rudder $\delta$ is shown. 
Notice the smooth behaviour of $\delta(t)$ even for relatively large wave disturbances. The autopilot does not compensate for the 1st-order wave disturbances.

\section{Case 4: PID-control with wave filtering}

Figure 7 shows the time-response of the ship when the notch filter in (34) is applied to the yaw angle measurements and the reference model is in the form (31) with $T_{i}=5 / \omega_{0}$. Both rudder offset and 1st-order wave disturbances are introduced. The initial values of $\Theta(t)$ and $\boldsymbol{P}(t)$ are the final values from a simulation with step inputs and

$$
\hat{\lambda}(s)=\lambda_{0}(s) n_{m}(s)=(s+1)^{3}\left(s+1 / T_{i}\right)^{2}
$$

It is seen that excellent tracking is obtained despite the noisy measurement and the rudder offset.

\section{Conclusions}

In this paper a globally stable adaptive autopilot has been presented. Only output feedback in terms of compass measurements was used. A cascaded notch filter has been included in the design to obtain proper wave disturbance filtering whereas integral action is obtained by applying a reference model shaping technique. The adaptive autopilot applied to the ship with a cascaded notch filter proved to be easy to tune even with no a priori information about the ship. Worst case initial values on $\Theta(t)$ may imply large tracking errors, but one step in the reference signal is usually sufficient to improve the performance significantly. In addition the 1st-order wave disturbances did introduce parameter drift. The MRAC also appeared to be robust for rudder saturation.

\section{ACKNOWLEDGEMENT}

This work was sponsored by the Center of Maritime Research at the Department of Engineering Cybernetics, NTH and SINTEF Automatic Control.

\section{Appendix A. Ship parameters}

The following ship parameters corresponding to a container ship with length 161 meters are taken from p. 174 in Fossen (1994)

$$
\begin{array}{ll}
T_{1}=18 \cdot 5, & T_{2}=7.8 \\
T_{3}=118.0, & K=0.185
\end{array}
$$

\section{Appendix B. Stability proof}

The stability proof is given by Sastry and Bodson (1989). Consider a SISO, LTI transfer function

$$
\frac{\psi_{p}}{\delta}(s)=K_{p} \frac{n_{p}(s)}{d_{p}(s)}
$$

which is minimum phase and where $K_{p}>0$. In addition $n_{p}(s)$ and $d_{p}(s)$ are coprime, monic and with degree $m$ and $n$, respectively, where $\gamma_{p}=n-m>0$. Also consider a SISO, LTI reference model

$$
M(s)=K_{m} \frac{n_{m}(s)}{d_{m}(s)}
$$

which is minimum phase, stable and with relative degree $\gamma_{m}=\gamma_{p}$. In addition $K_{m}>0$, $n_{m}(s)$ and $d_{m}(s)$ are coprime and monic. 
Let the controller be chosen as

$$
\delta(s)=\Theta^{\mathrm{T}}(s) w(s)
$$

where $\Theta(s), w(s) \in \mathbb{R}^{2 n-2}$ and the two signal vectors are defined as

$$
\begin{gathered}
\boldsymbol{w}_{1}(s)=\frac{1}{\lambda(s)}\left[\begin{array}{c}
1 \\
\vdots \\
s^{n+1}
\end{array}\right] \delta(s) \\
\boldsymbol{w}_{2}(s)=\frac{1}{\lambda(s)}\left[\begin{array}{c}
1 \\
\vdots \\
s^{n+2}
\end{array}\right] \psi_{p}(s)
\end{gathered}
$$

where $\lambda(s)$ has dimension $n-1$ and is Hurwitz. Then $w(s)$ is given by

$$
\boldsymbol{w}(s)=\left[\begin{array}{c}
r(s) \\
\boldsymbol{w}_{1}(s) \\
\psi_{p}(s) \\
\boldsymbol{w}_{2}(s)
\end{array}\right]
$$

where $r(s)$ is the reference signal. $\Theta(s)$ is given by

$$
\begin{gathered}
\boldsymbol{\Theta}(s)=-g \frac{e_{2}(s) \boldsymbol{P}(s) \boldsymbol{v}(s)}{s\left[1+\gamma \boldsymbol{v}^{\mathrm{T}}(s) \boldsymbol{P}(s) \boldsymbol{v}(s)\right]} \\
\boldsymbol{P}(s)=-g \frac{\boldsymbol{P}(s) \boldsymbol{v}(s) \boldsymbol{v}^{\mathrm{T}}(s) \boldsymbol{P}(s)}{s\left[1+\gamma \boldsymbol{v}^{\mathrm{T}}(s) \boldsymbol{P}(s) \boldsymbol{v}(s)\right]}
\end{gathered}
$$

where $g, \gamma \in \mathbb{R}^{+}, \boldsymbol{P}(s) \in \mathbb{R}^{(2 n-2) \times(2 n-2)}, e_{2}(s)=\Theta^{\mathrm{T}}(s) v(s)-L^{-1}(s) \delta(s), L^{-1}(s)$ is a stable, minimum phase transfer function with relative degree $\gamma_{l}=n-m$ and

$$
v(s)=L^{-1}(s)\left[\begin{array}{c}
M^{-1}(s) \psi_{p}(s) \\
w_{1}(s) \\
\psi_{p}(s) \\
w_{2}(s)
\end{array}\right]
$$

Then, according to Sastry and Bodson (1989) the controller (59) will stabilize the transfer function (57) and $\psi_{p}(t) \rightarrow \psi_{m}(t)$ as $t \rightarrow \infty$ if $r(t)$ and $c_{0}(t)$ satisfy the following two conditions:

1. Reference input assumption:

The reference signal, $r(t)$, is piecewise continuous and bounded on $\mathbb{R}^{+}$.

2. Bound on gain:

An upper bound on the gain $K_{p}, K_{\max }$, exist and is known. Then

$$
\begin{gathered}
K_{p} \leqslant K_{\max } \\
c_{\min } \triangleq K_{m} / K_{\max }
\end{gathered}
$$


and $c_{0}(t)$ has to satisfy conditions (a) and (b):

(a) $c_{0}(0) \geqslant c_{\min }$

(b) If $c_{0}(t) \leqslant c_{\min }$ and $c_{0}(t) \leqslant 0$ then $\dot{c}_{0}(t):=0$

See Sastry and Bodson (1989) for details on the proof.

\section{REFERENCES}

BAlChEN, J. G., JensSEN, N. A. and SÆLID, S. (1976). Dynamic positioning using Kalman filtering and optimal control theory. In: IFACIIFIP Symposium on Automation in Offshore Oil Field Operation. Holland, Amsterdam. pp. 183-186.

Balchen, J. G., JenSSEN, N. A. and SÆLID, S. (1980a). Dynamic positioning of floating vessels based on Kalman filtering and optimal control. In: Proceedings of the 19th IEEE Conference on Decision and Control. New York, NY. pp. 852-864.

BALChEN, J. G., JenSSEN, N. A., MATHISEN, E. and S Flid, S. (1980b). Dynamic positioning system based on Kalman filtering and optimal control. Modeling, Identification and Control, 1, 135-163.

Bodson, M. (1986). Stability, Convergence and Robustness of Adaptive Systems. PhD thesis. University of California, Berkeley.

BoDSON, M. and SASTRY, S. (1987). Input error versus output error model reference adaptive control. In: Proceedings of the 1987 American Control Conference. Minneapolis, USA, pp. 224-229.

Fossen, T. I. (1994). Guidance and Control of Ocean Vehicles (John Wiley and Sons Ltd).

Grimble, M. J., Patton, R. J., and Wise, D. A. (1980a). The design of dynamic positioning control systems using stochastic optimal control theory. Optimal Control Applications and Methods, 1, 167-202.

Grimble, M. J., PATTON, R. J. and Wise, D. A. (1980b). Use of Kalman filtering techniques in dynamic ship positioning systems. IEE Proceedings, D, 127, 93-102.

HolzhÜTER, T. and STRAuCH, H. (1987). A commercial adaptive autopilot for ships: design and experimental experience. In: Proceedings of the 10th IFAC World Congress. July 27-31, Munich, Germany. pp. 226-230.

Nomoto, K., TAGUCHI, T., Honda, K. and Hirano, S. (1957). On the steering qualities of ships. Technical report. International Shipbuilding Progress, Vol. 4.

Reid, R. E., Tugcu, A. K., and Mears, B. C. (1984). The use of wave filter design in Kalman filter state estimation of the automatic steering problem of a tanker in a seaway. IEEE Transactions on Automatic Control, 29, 577-584.

SALID, S. and JENSSEN, N. A. (1983). Adaptive ship autopilot with wave filter. Modeling, Identification and Control, 4, 33-46.

SAStry, S. and Bodson, M. (1989). Adaptive Control. (Prentice-Hall Int. Editions. New Jersey). 